\title{
Lettre à la rédaction : Une nouvelle souche de chanvre à fibres sans cannabinoïdes
}

\section{Letter to the editor: \\ A new cannabinoids-free fibre hemp strains}

\section{Gilbert FOURNIER $^{(1) *}$, Jocelyne BAUSSET ${ }^{(1)}$, Olivier BEHEREC ${ }^{(2)}$, Sylvestre BERTUCELLI ${ }^{(2)}$}

(1) Laboratoire de Pharmacognosie, Faculté de Pharmacie, UMR 8076 CNRS (BioCIS), 5, rue J.-B. Clément F-92290 CHÂTENAY-MALABRY

(2) Fédération Nationale des Producteurs de Chanvre, 20, rue Paul Ligneul - F-72000 LE MANS

*Auteur à qui adresser la correspondance : Professeur Gilbert FOURNIER, Laboratoire de Pharmacognosie, Faculté de Pharmacie, UMR 8076 CNRS (BioCIS), 5, rue J.-B. Clément - F-92296 CHÂTENAY-MALABRY Tél : 0143835597 - Fax : 0146835710 - E-mail : gilbert.fournier@cep.u-psud.fr

(Reçu le 19 avril 2005 ; accepté après modifications le 5 juillet 2005)

Les cannabinoïdes sont des substances spécifiques du chanvre, Cannabis sativa L. Plus d'une soixantaine sont connus (1), notamment le cannabichromène (CBC), le cannabidiol (CBD) et le cannabigérol (CBG), précurseur biogénétique du $\triangle-9-\mathrm{THC}(2-8)$ selon le schéma suivant:

acide olivétolique + pyrophosphate de géranyle

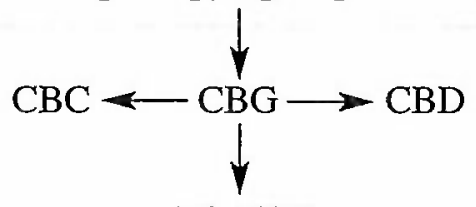

$\triangle-9-\mathrm{THC}$
Le $\triangle-9$-THC présente des propriétés psychotropes, ce qui n'est pas le cas pour le CBD et le CBG. Les réglementations relatives au chanvre sont d'ailleurs liées à la seule présence du $\triangle-9-T H C$. Selon les proportions relatives de $\Delta-9-T H C$ et de $\mathrm{CBD}$, il est désormais classique de distinguer les chimiotypes «Drogue» $(\Delta-9-T H C \gg$ $\mathrm{CBD}$ ), «Drogue intermédiaire» (teneurs en $\triangle-9-\mathrm{THC}$ et en CBD à peu près identiques) et «Fibres» $(\Delta-9$-THC $<<$ CBD) (9-15).

Selon plusieurs règlements publiés par le Journal officiel des Communautés européennes, la culture d'une vingtaine de variétés de chanvre est aujourd'hui autorisée (16). La plupart de ces variétés sont monoïques; elles se caractérisent toutes par des teneurs en $\Delta-9$ - 
THC inférieures à $0,2 \%$. Il s'agit de variétés appartenant au chimiotype «Fibres». Deux autres variétés autorisées, dénommées Santhica 23 et Santhica 27, ont la particularité de ne pas renfermer de $\Delta-9$-THC $\left(<10^{-5} \%\right)$, ni de CBD. La biosynthèse des cannabinoïdes est quasiment arrêtée au stade du CBG. Ce type chimique avait été décrit par l'un de nous en 1987 (17). L'intérêt de ces nouvelles variétés est important en terme de santé publique puisqu'elles ne présentent aucune propriété psychoactive. Par ailleurs, les caractéristiques agronomiques et les critères de qualité de ces variétés (notamment leurs rendements en fibres corticales) sont comparables à ceux des autres variétés industrielles autorisées. Il avait été proposé que Santhica 23 et Santhica 27, compte tenu de leurs utilisations et du fait de leur composition en cannabinoïdes, devraient désormais être considérées comme les premières «variétés à fibres de seconde génération» (18).

Depuis la découverte de ces nouvelles variétés (Santhica 23 et Santhica 27), et lors des nombreuses analyses que nous effectuons chaque année dans le cadre de programmes de sélection, nous avons eu l'opportunité de repérer des individus ne comportant aucun cannabinoïde (avec donc notamment absence de $\Delta-9$ THC, de CBD et de CBG). Il semble que, dans ce cas, la biogenèse de ces constituants se soit arrêtée encore plus tôt, probablement du fait de l'absence de l'enzyme catalysant la condensation du pyrophosphate de géranyle avec l'acide olivétolique (7).

Deux fois par semaine, nous avons analysé ces individus tout au long de leur développement (depuis le début de la végétation jusqu'à la complète maturation des graines) ; à aucun moment il n'a été possible de détecter la présence de cannabinoïdes $\left(<10^{-5} \%\right)$. Ce suivi a été effectué par chromatographie en phase gazeuse (détecteur à ionisation de flamme : DIF) selon la méthode communautaire pour la détermination quantitative du $\triangle-9-T H C$ des variétés de chanvre décrite en annexe de l'arrêté du 24 février 2004 paru au Journal officiel de la République française le 21 mars 2004 (19).

Cette nouvelle souche, présentant ce type chimique inédit et ayant une morphologie et une vigueur comparables à celles des variétés dont la culture est autorisée, pourrait conduire à la création d'une nouvelle «variété à fibres de troisième génération». Des expérimentations sont encore en cours afin d'éprouver sa productivité en fibres. Par ailleurs, divers essais préliminaires ont été menés sur l'huile des graines et sur l'huile essentielle des feuilles : comparativement aux autres variétés classiquement cultivées en France, aucune différence significative'n'a été constatée tant quantitativement que qualitativement pour ces deux catégories de substances. La création d'une variété stabilisée à partir de cette souche est en cours ; elle sera disponible dès qu'elle sera inscrite au catalogue des variétés.

Le tableau I résume les proportions des principaux cannabinoïdes présentés par les différents types chimiques ici évoqués.

\section{Conclusion}

En Europe, la culture du chanvre est règlementairement autorisée avec des variétés dont la teneur en $\triangle$-9-THC est inférieure à $0,2 \%$. Depuis quelques années deux nouvelles variétés (Santhica 23 et Santhica 27) peuvent également être cultivées : elles ne renferment pas de $\triangle$-9-THC. La biogenèse des cannabinoïdes s'est arrêtée au stade du CBG, précurseur du CBD et du $\triangle-9-\mathrm{THC}$. Une nouvelle souche est ici présentée : elle ne renferme plus de cannabinoïdes ; la biogenèse s'est arrêtée au stade des précurseurs des cannabinoïdes.

Tableau I : Proportions des principaux cannabinoüdes présents dans divers types chimiques du chanvre.

\begin{tabular}{|c|c|c|c|c|c|}
\hline \multirow[b]{2}{*}{ Cannabinoïdes } & \multirow[b]{2}{*}{ "Drogue" } & \multirow[b]{2}{*}{ "Drogue intermédiaire" } & \multicolumn{3}{|c|}{ sqbres" } \\
\hline & & & $\begin{array}{l}\text { 1'ro génération } \\
\text { (Epsilon, Fedora, Felina, } \\
\text { Ferimon, Futura,..) }\end{array}$ & $\begin{array}{l}2 \text { génération } \\
\text { (Santhica) }\end{array}$ & 3 génération \\
\hline$\triangle-9-$ THC & $t+t+$ & +++ & $+(<0,2 \%)$ & - & - \\
\hline CBD & \pm & $t+t$ & $t+t$ & \pm & $\therefore-$ \\
\hline CBG & \pm & \pm & \pm & $+t+$ & - \\
\hline
\end{tabular}




\section{Références}

1. Turner C.E., Elsohly M.A., Boeren E.G. Constituents of Cannabis sativa L. XVII: A review of the natural constituents. J. Nat. Prod. 1980; 43 : 169-234.

2. Shoyama Y., Yagi M., Nishioka I., Yamauchi T. Biosynthesis of cannabinoid acids. Phytochemistry 1975 ; 14 : 2189-92.

3. Kajima M., Piraux M. The biogenesis of cannabinoids in Cannabis sativa. Phytochemistry $1982 ; 21$ : 67-9.

4. Hanus L. Biogenesis of cannabinoid substances in the plant. Acta Univ. Olomuc., Fac. Med. 1987 ; 116 : 47-53.

5. Morimoto S., Taura F., Shoyama Y. Biosynthesis of cannabinoids in Cannabis sativa L. Current Topics in Phytochemistry $1999 ; 2$ : 103-13.

6. Fellermeier M., Eisenreich W., Bacher A., Zenk M.H. Biosynthesis of cannabinoids. Incorporation experiments with ${ }^{13} \mathrm{C}$-labelled glucoses. Eur. J. Biochem. $2001 ; 268$ : 1596-604.

7. Fellermeier M., Zenk M.H. Prenylation of olivetolate by a hemp transferase yields cannabigerolic acid, the precursor of tetrahydrocannabinol. FEBS Lett. 1998; $427: 283-5$.

8. de Meijer E..P.M., Bagatta M., Carboni A., Crucitti P., Moliterni V.M.C., Ranalli P., Mandolino G. The inheritance of chemical phenotype in Cannabis sativa L. Genetics $2003 ; 163: 335-46$.

9. Fournier G., Paris M. Détermination de chimiotypes à partir des cannabinoïdes chez le chanvre à fibres monoïque (Cannabis sativa L.). Possibilités de sélection. Physiol. Vég. $1980 ; 18$ : 349-56.

10. Fetterman P.S., Keith E.S., Waller C.W., Guerrero O., Doorenbos N.J., Quimby M.W. Mississippi-Grown Cannabis sativa L.: preliminary observation on chemical definition of phenotype and variations in tetrahydrocannabinol content versus age, sex, and plant part. J. Pharm. Sci. $1971 ; 60: 1246-9$.
11. Small E. The species problem in Cannabis : science \& semantics. Toronto: Corpus, 1979 ; I-218p, II-156p.

12. Turner C.E., Elsohly M.A., Cheng P.C., Lewis G. Constituents of Cannabis sativa L., XIV : Intrinsic problems in classifying cannabis based on a single cannabinoid analysis. J. Nat. Prod. 1979 ; 42 : 317-19.

13. Fournier G. Les chimiotypes du chanvre (Cannabis sativa L .). Intérêt pour un programme de sélection. Agronomie $1981 ; 1: 679-88$.

14. Brenneisen R., Elsohly M.A. Chromatographic and spectroscopic profiles of Cannabis of different origins : Part I. J. Forensic Sci. 1988 ; 33 : 1385-404.

15. Fournier G. La sélection du chanvre à fibres (Cannabis sativa L.) en France. Chanvre et THC. C. R. Acad. Agric. Fr. $2000 ; 86: 209-17$.

16. Journal Officiel des Communautés européennes Règlement (CE) $N^{\circ} 436 / 2005$ de la Commission du 17 mars 2005, modifiant le règlement (CE) $n^{\circ} 796 / 2004$.

17. Fournier G., Richez-Dumanois C., Duvezin J., Mathieu J.P., Paris M. Identification of a new chemotype in Cannabis sativa : cannabigerol-dominant plants, biogenetic and agronomic prospects. Planta Med. 1987 ; 53 : 277 80.

18. Fournier G., Béhérec O., Bertucelli S. Santhica 23 et 27 : deux variétés de chanvre (Cannabis sativa L.) sans $\Delta-9$ THC. Ann. Toxicol. Anal. 2004 ; 16 : 128-32.

19. Journal Officiel de la République française - Arrêté du 24 février 2004 modifiant l'arrêté du 22 août 1990 portant application de l'article R.5181 du code de la santé publique pour le cannabis - J.O. du 21 mars 2004. 